\section{Epidemiologia da sífilis gestacional em Fortaleza, Ceará, Brasil: um agravo sem controle}

\author{
Epidemiology of gestational syphilis \\ in Fortaleza, Ceará State, Brazil: \\ an uncontrolled disease
}

\author{
1 Universidade de Fortaleza, \\ Fortaleza, Brasil. \\ Correspondência \\ A. L. A. Campos \\ Universidade de Fortaleza. \\ Av. Washington Soares 1321, \\ Fortaleza, CE 60811-905, \\ Brasil. \\ ninaacampos@yahoo.com.br
}

\begin{abstract}
This cross-sectional study investigated the epidemiological profile of pregnant women with positive VDRL in Fortaleza, Ceará State, Brazil, in 2008. The study verified the proportion of pregnant women with syphilis that was classified as treated incorrectly according to Brazilian Ministry of Health guidelines, and assessed the reasons for inadequate treatment. Fifty-eight women who had given birth at five public maternity hospitals were interviewed consecutively following delivery. Data were also recovered from medical files and pregnancy cards. Sociodemographic and obstetric data and information related to the diagnosis and treatment of syphilis in both pregnant women and their partners were analyzed. Only three (5.2\%; 95\%CI: 1.8\%-14.1\%) pregnant women had received adequate treatment. The main reason for inadequate treatment was lack of partner treatment (88\% of cases; 95\%CI: 76.2\%-94.4\%). Medical care as currently provided does not guarantee the control of gestational syphilis in this sample.

Congenital Syphilis; Pregnant Women; Prenatal Care
\end{abstract}

Ana Luiza de Araujo Campos 1

Maria Alix Leite Araújo 1

Simone Paes de Melo 1

Marcelo Luiz Carvalho Gonçalves 1

\section{Introdução}

A sífilis gestacional, apesar de apresentar diagnóstico simples e tratamento eficaz, ainda apresenta prevalência alarmante, principalmente em países pobres ou em desenvolvimento $1,2,3$. O risco de transmissão vertical da sífilis varia de $30 \%$ a 100\%, dependendo da fase clínica da doença na gestante. Em aproximadamente $40 \%$ das infecções intra-uterinas não tratadas ocorre o aborto espontâneo ou a morte perinatal 4 .

Acredita-se que ocorram anualmente 12 milhões de casos novos de sífilis na população adulta em todo o mundo, 90\% deles nos países em desenvolvimento ${ }^{5}$. As estimativas apontam a sífilis congênita como responsável por mais de 500 mil mortes fetais por ano no mundo ${ }^{3}$. Na região da América Latina e Caribe, a prevalência da sífilis nos recém-nascidos é de $3,1 \%$, oscilando entre $1 \%$ no Peru e $6,2 \%$ no Paraguai 6 . Na Bolívia, a prevalência de sífilis gestacional é de $7,2 \%$, com taxa de transmissão vertical de 15,7\% 7. Estimase que no Brasil a prevalência média da sífilis em parturientes varie entre $1,4 \%$ e $2,8 \% 4$, com uma taxa de transmissão vertical de $25 \% 1$.

Apesar da magnitude, esses números estão subestimados, pois a falta de notificação é uma realidade em muitos países. Na América Latina, estima-se a subnotificação de $34 \%$ no Peru, $32,2 \%$ na Argentina, $26 \%$ no Chile e $22,2 \%$ na Venezuela 5 . No Brasil, apesar da sífilis congênita e a sífilis na gestante serem agravos de notificação 
compulsória desde 1986 e 2005, respectivamente 8 , apenas $32 \%$ dos casos de sífilis gestacional e $17,4 \%$ de sífilis congênita são notificados 1,8. Esses dados refletem deficiências importantes na qualidade dos serviços da assistência no período pré-natal e no parto 8,9 .

Paradoxalmente, a sífilis é uma doença sexualmente transmissível que pode ser facilmente controlada, levando-se em consideração a existência de testes diagnósticos sensíveis, tratamento eficaz e de baixo custo ${ }^{1}$. A ação mais consistente para controle da sífilis congênita está na garantia de uma assistência pré-natal ampla e de qualidade, garantindo-se o diagnóstico precoce $\mathrm{e}$ o tratamento em tempo hábil 10,11.

Os fundamentos centrais do controle da sífilis congênita estão baseados na triagem sorológica das gestantes por meio de testes não treponêmicos, principalmente o Venereal Disease Research Laboratory (VDRL), e o tratamento adequado das gestantes e de seus parceiros sexuais 10,11,12,13.

Este estudo teve por objetivo verificar o percentual das gestantes com exame de VDRL reagente em qualquer período gestacional, ou na ocasião do parto, que não foram adequadamente tratadas, buscando identificar os motivos da não adequação do tratamento e apresentar o perfil sociodemográfico, obstétrico e epidemiológico destas gestantes.

\section{Metodologia}

Trata-se de um estudo transversal e descritivo, realizado em cinco maternidades públicas localizadas no Município de Fortaleza, Ceará, Brasil: (i) Hospital Distrital Nossa Senhora da Conceição, (ii) Hospital Distrital Gonzaga Mota - Messejana, (iii) Hospital Distrital Gonzaga Mota - Barra do Ceará, (iv) Hospital Distrital Gonzaga Mota - José Walter e (v) Hospital Geral Dr. César Cals. Os quatro primeiros sob gestão municipal e o último, estadual.

Foram convidadas a participar desta pesquisa todas as gestantes admitidas nas cinco maternidades para a realização do parto entre os meses de maio e outubro de 2008, e que tiveram o exame sorológico de VDRL reagente por ocasião do parto. Também foram incluídas as que não fizeram o exame no momento do parto, mas que apresentaram VDRL reagente em qualquer momento do período gestacional, condição identificada no cartão da gestante.

Entre as gestantes admitidas nas maternidades, 60 preencheram os critérios de inclusão e foram convidadas a participar do estudo, após esclarecimentos sobre os objetivos e metodologia da pesquisa. Dessas, 58 aceitaram participar e só foram incluídas no estudo após assinarem o Termo de Consentimento Livre e Esclarecido. Em seguida, foi aplicado às participantes um questionário com perguntas estruturadas, referentes a variáveis sociodemográficas, obstétricas e as relacionadas à infecção por Treponema pallidum e ao tratamento recebido. Os dados foram complementados com as informações disponíveis no cartão da gestante e no seu prontuário.

Foi considerado tratamento materno adequado todo tratamento completo e adequado à fase da doença, realizado com penicilina e finalizado pelo menos 30 dias antes do parto, tendo sido o parceiro concomitantemente tratado, de acordo com as normas do Ministério da Saúde para prevenção da transmissão vertical da sífilis 1 .

Considerando a ocorrência de 40 mil partos por ano no município (Departamento de Informática do SUS. Indicadores de dados básicos do Brasil. Indicadores de cobertura. http:// w3.datasus.gov.br, acessado em 13/Out/2008), freqüência estimada de $90 \%$ de gestantes não adequadamente tratadas 1 , precisão de $10 \%$ e nível de 95\% de confiança, a amostra mínima necessária a ser avaliada foi de 35 gestantes. Foram entrevistadas gestantes em quantidade superior ao número amostral mínimo. Os dados foram tabulados e analisados no programa SPSS (SPSS Inc., Chicago, Estados Unidos). As variáveis foram submetidas à análise univariada por meio de distribuições de freqüências ou, quando aplicável, de medidas de tendência central e de dispersão. Os intervalos de 95\% de confiança foram calculados pelo método de Wilson. O cálculo amostral foi realizado com o auxílio do programa Epi Info 3.5.1 (Centers for Disease Control and Prevention, Atlanta, Estados Unidos).

Esta pesquisa foi aprovada pelo Comitê de Ética da Universidade de Fortaleza com o parecer número 059/2008, de acordo com a Resolução $n^{o}$. 196/96 do Conselho Nacional de Saúde. Assegurou-se o sigilo das informações em todas as etapas do estudo.

\section{Resultados}

No período de seis meses do estudo foram entrevistadas 58 gestantes com VDRL reagente no parto ou durante a assistência pré-natal. A média da idade foi de 23,8 anos, variando de 14 a 39 anos (DP: 5,8). A Tabela 1 mostra a distribuição das gestantes com VDRL reagente, de acordo com as variáveis sociodemográficas, além da situação e tempo de relacionamento com o pai do recémnascido. A Tabela 2 apresenta a distribuição das gestantes de acordo com as variáveis obstétricas avaliadas, e a Tabela 3 mostra a distribuição das 


\begin{tabular}{|c|c|c|c|}
\hline Variáveis & Média (DP) & $\mathbf{n}$ & $\%$ \\
\hline Faixa etária (em anos) & $23,8(5,8)$ & & \\
\hline $11-20$ & & 20 & 34,5 \\
\hline $21-30$ & & 31 & 53,4 \\
\hline $31-40$ & & 7 & 12,1 \\
\hline Escolaridade (em anos) & $6,2(3,6)$ & & \\
\hline 0 & & 4 & 6,9 \\
\hline $1-3$ & & 11 & 19,0 \\
\hline $4-7$ & & 23 & 39,7 \\
\hline $8-11$ & & 19 & 32,8 \\
\hline$\geq 12$ & & 1 & 1,7 \\
\hline \multicolumn{4}{|l|}{ Estado civil } \\
\hline Casada & & 6 & 10,3 \\
\hline União estável & & 33 & 56,9 \\
\hline Separada & & 2 & 3,4 \\
\hline Solteira & & 17 & 29,3 \\
\hline Renda familiar (em salários mínimos) & $0,9(0,7)$ & & \\
\hline$<1$ & & 37 & 63,8 \\
\hline $1-2$ & & 19 & 32,8 \\
\hline $3-4$ & & 2 & 3,4 \\
\hline \multicolumn{4}{|l|}{ Parceiro atual é o pai do recém-nascido } \\
\hline Sim & & 52 & 89,7 \\
\hline Não & & 2 & 3,4 \\
\hline Ignorado & & 4 & 6,9 \\
\hline Tempo de relacionamento com o pai do recém-nascido (em anos) & $3,1(2,8)$ & & \\
\hline$<1$ & & 11 & 19,0 \\
\hline $1-5$ & & 28 & 48,3 \\
\hline$>5$ & & 13 & 22,4 \\
\hline Sem relacionamento & & 6 & 10,3 \\
\hline
\end{tabular}

gestantes de acordo com as variáveis relacionadas à sífilis. A Tabela 4 relaciona o percentual de adequação do tratamento das gestantes e dos parceiros, e os motivos conhecidos de inadequação do tratamento recebido pela gestante.

Em 14 (24,1\%) casos as gestantes informaram que os parceiros foram tratados, em $38(65,5 \%)$ não houve tratamento do parceiro e seis (10,3\%) não souberam informar. Entre os 14 casos mencionados de tratamento do parceiro, em apenas seis $(42,9 \%)$ foi relatado tratamento completo, correspondendo a $10,3 \%$ da amostra total.

Entre as 29 gestantes nas quais o diagnóstico de sífilis foi feito durante o pré-natal, nove (31\%; IC95\%: 17,3\%-49,2\%) tiveram o tratamento finalizado 30 dias ou mais antes do parto, em 11 (37,9\%; IC95\%: 22,7\%-56,0\%) o tratamento foi finalizado em menos de 30 dias da data do parto, em um (3,4\%; IC95\%: 0,6\%-17,2\%) não foi realizado o tratamento e em oito $(27,6 \%$; IC95\%: $14,7 \%-45,7 \%$ ) não foi possível determinar a data do término do tratamento. Das 28 gestantes tratadas, sete (25\%) relataram 2,4 milhões de UI de penicilina benzatina, cinco (17,9\%) 4,8 milhões de UI, 15 (53,6\%) 7,2 milhões de UI e uma (3,6\%) gestante 9,6 milhões de UI de penicilina benzatina. Não foi possível verificar a fase da doença das 58 gestantes. Entre todas elas não há qualquer registro da realização de controle de cura.

Foram consideradas adequadamente tratadas apenas três (5,2\%; IC95\%: 1,8\%-14,1\%) gestantes, sendo que duas tiveram nove consultas de pré-natal e uma apenas quatro consultas. Duas dessas gestantes iniciaram o pré-natal no primeiro trimestre gestacional e a terceira no segundo trimestre. Todas as três foram tratadas 
Tabela 2

Distribuição das gestantes com VDRL reagente na gestação e/ou no momento do parto, de acordo com variáveis obstétricas. Fortaleza, Ceará, Brasil, $2008(n=58)$.

\begin{tabular}{|c|c|c|c|}
\hline Variáveis & Média (DP) & $\mathbf{n}$ & $\%$ \\
\hline \multicolumn{4}{|l|}{ Realização de pré-natal } \\
\hline Sim & & 47 & 81,0 \\
\hline Não & & 11 & 19,0 \\
\hline Número de consultas de pré-natal & $4,2(3,0)$ & & \\
\hline 0 & & 11 & 19,0 \\
\hline $1-5$ & & 23 & 39,6 \\
\hline$\geq 6$ & & 24 & 41,4 \\
\hline Número de consultas de pré-natal com médico & $2,9(2,2)$ & & \\
\hline 0 & & 13 & 22,4 \\
\hline 1 & & 2 & 3,5 \\
\hline$\geq 2$ & & 37 & 63,8 \\
\hline Ignorado & & 6 & 10,3 \\
\hline \multicolumn{4}{|l|}{ Início do pré-natal ( $n=47)$} \\
\hline 1ㅇ trimestre gestacional & & 22 & 46,8 \\
\hline 2ㅇ trimestre gestacional & & 21 & 44,7 \\
\hline 3o trimestre gestacional & & 4 & 8,5 \\
\hline Idade gestacional no parto (em semanas) & $36,7(4,4)$ & & \\
\hline$<37$ & & 21 & 36,2 \\
\hline $37-42$ & & 33 & 56,9 \\
\hline$>42$ & & 1 & 1,7 \\
\hline Ignorado & & 3 & 5,2 \\
\hline \multicolumn{4}{|l|}{ Primiparidade } \\
\hline Sim & & 22 & 37,9 \\
\hline Não & & 36 & 62,1 \\
\hline \multicolumn{4}{|l|}{ Natimorto (gestação atual) } \\
\hline $\operatorname{Sim}$ & & 1 & 1,7 \\
\hline Não & & 57 & 98,3 \\
\hline \multicolumn{4}{|l|}{ Número de abortos espontâneos } \\
\hline 0 & & 42 & 72,4 \\
\hline 1 & & 14 & 24,1 \\
\hline 2 & & 2 & 3,5 \\
\hline \multicolumn{4}{|c|}{ Número de natimortos em gestações anteriores $(n=36)$} \\
\hline 0 & & 31 & 86,1 \\
\hline 1 & & 5 & 13,9 \\
\hline \multicolumn{4}{|l|}{ Cartão da gestante } \\
\hline Devidamente preenchido & & 16 & 27,6 \\
\hline Não preenchido devidamente & & 23 & 39,7 \\
\hline Não possui & & 17 & 29,3 \\
\hline Ignorado & & 2 & 3,4 \\
\hline
\end{tabular}

com penicilina benzatina na dose de 7,2 milhões de UI.

Das 32 gestantes que tiveram o VDRL reagente durante o período pré-natal, $30(51,7 \%)$ tinham titulação conhecida, variando entre 1:2 e 1:128, com mediana e moda de 1:8. O VDRL foi reagen- te na ocasião do parto em $55(94,8 \%)$ gestantes. Nesse grupo, a titulação desse exame foi conhecida em $51(87,9 \%)$ gestantes, variando entre 1:1 e 1:64, com mediana de 1:8 e moda de 1:16.

Foi possível realizar a comparação entre os títulos de VDRL realizado no pré-natal com 
Tabela 3

Distribuição das gestantes com VDRL reagente na gestação e/ou no momento do parto, de acordo com variáveis relacionadas à sífilis. Fortaleza, Ceará, Brasil, $2008(n=58)$.

\begin{tabular}{lcc}
\hline Variáveis & $\mathbf{n}$ & $\%$ \\
\hline Momento do diagnóstico da sífilis na & & \\
gestação atual & & \\
$\quad$ Durante o pré-natal & 29 & 50,0 \\
Parto/puerpério & 23 & 39,6 \\
Antes do pré-natal & 3 & 5,2 \\
Ignorado & 3 & 5,2 \\
VDRL no pré-natal & & \\
Reagente & 32 & 55,2 \\
Não reagente & 4 & 6,9 \\
Não realizado & 7 & 12,0 \\
Ignorado & 15 & 25,9 \\
VDRL no parto & & \\
Reagente & 55 & 94,8 \\
Não realizado & 2 & 3,5 \\
Ignorado & 1 & 1,7
\end{tabular}

o exame realizado por ocasião do parto em 19 $(32,7 \%)$ gestantes, considerando-se um tempo mínimo de um mês entre os dois exames. Em duas $(10,5 \%)$ dessas 19 gestantes houve aumento dos títulos de VDRL em pelo menos quatro vezes ou duas diluições. Uma delas foi medicada com 2,4 milhões de UI de penicilina benzatina durante o pré-natal e informou que o parceiro recebeu igual tratamento. A outra gestante foi medicada com 4,8 milhões de UI de penicilina benzatina durante o pré-natal e informou que o parceiro não foi tratado.

Considerando-se as 58 gestantes, observou-se que quatro $(6,9 \%)$ delas com VDRL não reagente no pré-natal apresentaram resultado reagente no momento do parto. Dentre as 47 gestantes que realizaram pré-natal, sete $(14,9 \%)$ não realizaram o VDRL nesse período.

\section{Discussão}

O perfil sociodemográfico das gestantes neste estudo indica que a sífilis está ocorrendo em jovens, com algum grau de escolaridade, renda familiar menor do que um salário mínimo e com parceria sexual fixa, resultados semelhantes aos de diversos autores 14,15,16,17,18,19. Observou-se um número elevado de adolescentes com sífilis gestacional, provavelmente refletindo o perfil das gestantes do município. Explicação seme- lhante pode ser aventada para a observação de que aproximadamente dois terços das gestantes com sífilis tinham renda familiar menor do que um salário mínimo.

Eram casadas ou mantinham união estável com o parceiro $39(67,2 \%)$ das gestantes, sendo que $52(89,7 \%)$ referiram o parceiro como pai do recém-nascido. A sífilis, nesse contexto, pode ser conseqüência de relacionamentos extraconjugais ou ter como fonte de infecção parceiros sexuais anteriores, já que $39(67,3 \%)$ gestantes referiram tempo de relacionamento com o atual parceiro igual ou inferior a cinco anos. Apesar da transmissibilidade da sífilis por contato sexual estar relacionada à fase da infecção, e declinar ao longo do tempo, é possível ocorrer infecção mesmo alguns anos após a lesão primária, o que teoricamente poderia justificar a presença de infecção entre parceiros monogâmicos.

A assistência pré-natal é fundamental à saúde materno-infantil. Nesse período, devem ser desenvolvidas atividades relacionadas à promoção da saúde e identificação de riscos para a gestante e o concepto, permitindo assim a prevenção de inúmeras complicações, além de reduzir ou eliminar fatores e comportamentos de risco associados a vários agravos à saúde 20,21,22. O Ministério da Saúde preconiza assistência de pré-natal com no mínimo seis consultas com profissionais de saúde, sendo pelo menos duas delas realizadas por médico. Aconselha ainda o início do atendimento no primeiro trimestre gestacional 23. Os dados oficiais apontam alta cobertura de prénatal no Brasil, onde quase $90 \%$ das gestantes são atendidas por profissionais qualificados 23 . Contudo, é importante salientar que ainda assim os resultados são insuficientes para o controle de vários agravos à saúde relacionados à gestação, não havendo redução significativa dos coeficientes de mortalidade materna e perinatal 24,25 .

À semelhança de outros estudos 10,15,22,26, a maior parte das gestantes $(81 \%)$ relatou a realização do acompanhamento pré-natal, e com início no primeiro trimestre de gestação em $46,8 \%$ dos casos. As duas consultas realizadas com o médico durante o pré-natal não asseguraram um desfecho mais favorável; 37 (63,8\%) das gestantes tiveram pelo menos duas consultas com este profissional durante o período pré-natal. Verificou-se, com base nos dados deste estudo e nos realizados por outros autores 10,15, que a garantia de um número mínimo de consultas de pré-natal não é suficiente para assegurar um atendimento de qualidade à gestante, o que torna as metas para o controle da sífilis congênita inalcançáveis com as práticas atuais.

Entre as gestantes que realizaram pré-natal, $22(46,8 \%)$ fizeram a primeira consulta no primei- 
Distribuição das gestantes com VDRL reagente na gestação e/ou no momento do parto, de acordo a adequação do tratamento recebido. Fortaleza, Ceará, Brasil, $2008(n=58)$.

\begin{tabular}{|c|c|c|c|}
\hline Variáveis & $\mathbf{n}$ & $\%$ & IC95\% \\
\hline \multicolumn{4}{|l|}{ Tratamento da gestante } \\
\hline Adequado & 3 & 5,2 & $1,8-14,1$ \\
\hline Não adequado\Não realizado & 50 & 86,2 & $76,1-93,5$ \\
\hline Ignorado & 5 & 8,6 & $3,7-18,6$ \\
\hline \multicolumn{4}{|l|}{ Adequação do tratamento do parceiro } \\
\hline $\operatorname{Sim}$ & 6 & 10,3 & $4,8-20,8$ \\
\hline Não & 46 & 79,4 & $67,2-87,7$ \\
\hline Ignorado & 6 & 10,3 & $4,8-20,8$ \\
\hline \multicolumn{4}{|c|}{ Motivos da inadequação do tratamento $(n=50)$ * } \\
\hline Não tratamento da gestante & 10 & 20,0 & $11,2-33,0$ \\
\hline Tratamento finalizado $<30$ dias do parto & 23 & 46,0 & $33,0-59,6$ \\
\hline Tratamento finalizado em prazo ignorado & 11 & 22,0 & $12,8-35,2$ \\
\hline Parceiro não adequadamente tratado & 44 & 88,0 & $76,2-94,4$ \\
\hline Parceiro com tratamento ignorado & 4 & 8,0 & $3,2-18,8$ \\
\hline
\end{tabular}

* Houve gestantes com mais de um motivo de inadequação do tratamento.

ro trimestre de gestação, $21(44,7 \%)$ no segundo trimestre e $4(8,5 \%)$ no terceiro, resultado semelhante ao encontrado por Araújo et al. 27, Silveira et al. 28 e Trevisan et al. 29. É de vital importância a utilização de marcadores da qualidade desse atendimento no planejamento, na gestão e na elaboração de políticas públicas relacionadas à saúde materno-infantil.

Realizaram pelo menos as seis consultas de pré-natal preconizadas pelo Ministério da Saúde $41,4 \%$ das gestantes pesquisadas, número próximo aos $46 \%$ encontrados por Saraceni \& Leal 30 no Município do Rio de Janeiro. Por outro lado, de acordo com os dados de Almeida \& Pereira 19, ao avaliar o perfil epidemiológico da sífilis congênita em Salvador (Bahia), apenas 12\% dos casos notificados realizaram mais de seis consultas de pré-natal. Essa diferença pode ser explicada pelas coberturas de pré-natal entre esses municípios avaliados. Outra explicação possível para essa discrepância pode estar relacionada ao tipo de estratificação realizada no número de consultas, maior de seis na pesquisa da Bahia, e seis ou mais neste estudo e no do Rio de Janeiro.

Quando a sífilis nas gestantes não é tratada, ou é inadequadamente tratada, pode ocasionar abortamentos, prematuridade e natimortalidade. Quando ocorre a infecção do concepto a partir de mães não tratadas, em aproximadamente $40 \%$ dos casos acontecem aborto espontâneo, natimorto ou óbito perinatal 4 . Neste estudo, $21(36,2 \%)$ recém-nascidos foram considerados prematuros (nascimento antes de 37 semanas de idade gestacional), valores ainda maiores que os encontrados por De Lorenzi \& Madi 16, em Caxias do Sul (Rio Grande do Sul), onde ocorreu prematuridade em $29,6 \%$ dos casos de sífilis gestacional.

Neste trabalho houve a ocorrência de um $(1,7 \%)$ caso de natimortalidade; embora provável, não foi possível determinar se a causa do óbito estava relacionada à sífilis congênita. Houve histórico de abortamento espontâneo prévio em $16(27,6 \%)$ gestantes. Esses números guardam grande proximidade com os encontrados por Saraceni et al. 9 e Almeida \& Pereira 19. Entre as $39(67,2 \%)$ gestantes multigestas, oito $(20,5 \%)$ referiram sífilis em gestação anterior, o que pode refletir tratamento inadequado naquela ocasião ou ainda uma reinfecção posterior.

Neste estudo foi observado que em 23 (39,7\%) gestantes o cartão não trazia as informações necessárias, e que em 17 (29,3\%) casos a gestante não possuía o cartão. Araújo et al. 27 e Lima 31 também evidenciam a falta de informações contidas em prontuários e cartões da gestante. Esse fato pode estar relacionado à falta de qualidade no atendimento pré-natal. O correto preenchimento do cartão da gestante é fundamental para que os profissionais de saúde possam realizar com segurança o seguimento do tratamento da sífilis na gestante.

De acordo com o preconizado pelo Ministério da Saúde, o exame de VDRL deve ser realizado 
logo no início da assistência pré-natal, repetido no terceiro trimestre gestacional e no momento do parto. Os dois primeiros exames visam a garantir o diagnóstico precoce à gestante com sífilis e seu tratamento em tempo hábil, e o terceiro permite o tratamento precoce da criança 32 . Apesar dessa recomendação, somente 32 (55,2\%) gestantes tiveram o diagnóstico ainda no período pré-natal. Não houve registro da realização do segundo VDRL nas gestantes. É importante ressaltar a necessidade da realização desse segundo exame por volta da $28^{\mathrm{a}}$ semana de gestação, conforme preconizado. Houve quatro $(6,9 \%)$ gestantes que apresentaram o único VDRL realizado no período pré-natal não reagente, tendo perdido a oportunidade de serem adequadamente tratadas caso o exame tivesse sido repetido no terceiro trimestre. Esses números demonstram a fragilidade do atendimento pré-natal, fato igualmente verificado por outros autores 18,29,30,33.

Para que a gestante com sífilis seja considerada adequadamente tratada, afastando a possibilidade de infecção do concepto, deve ser medicada com penicilina $G$ benzatina, nas doses apropriadas à fase da infecção, ter finalizado o tratamento pelo menos 30 dias antes do parto, $\mathrm{e}$ ter o parceiro concomitantemente tratado com o mesmo esquema terapêutico da gestante 12,23. Com as informações disponíveis neste estudo não foi possível estabelecer a fase clínica da infecção das gestantes. Dessa forma, a avaliação da adequação do tratamento foi feita mediante a data de finalização do esquema terapêutico utilizado e a realização do tratamento completo do parceiro. Considerando-se esses critérios, apenas três (5,2\%; IC95\%: 1,8\%-14,1\%) gestantes foram consideradas adequadamente tratadas. A principal causa de inadequação do tratamento foi a falta de tratamento do parceiro. Esses foram considerados devidamente tratados em apenas seis (10,6\%; IC95\%: 4,8\%-20,8\%) casos. Esses números estão bastante próximos dos encontrados em outros estudos em diferentes regiões do país $16,18,29,34$.

Este estudo evidenciou que a quantidade de consultas realizadas pela gestante em seu atendimento pré-natal não é suficiente para garantir o controle da sífilis. A diminuição significativa da sua incidência no período gestacional e a conseqüente diminuição de casos de sífilis congênita só poderá ser efetiva quando as medidas de prevenção e controle forem satisfatoriamente aplicadas 10,33. Para isso, é necessário que tanto os profissionais da saúde quanto os gestores estejam seriamente comprometidos com a qualidade dos serviços prestados na assistência prénatal. Considerando a importância dos registros referentes ao acompanhamento da gestante, cabe ressaltar a necessidade de melhoria nas informações registradas nos prontuários e nos cartões das gestantes.

\section{Resumo}

O objetivo deste estudo transversal foi conhecer o perfil epidemiológico das gestantes com VDRL reagente, em Fortaleza, Ceará, Brasil, no ano de 2008. Foi verificado o percentual das gestantes com sífilis que foram consideradas inadequadamente tratadas e os motivos da inadequação, de acordo com as normas do Ministério da Saúde. Foram entrevistadas 58 gestantes no pósparto imediato, internadas em cinco maternidades públicas do município, e consultadas as informações do prontuário e do cartão das gestantes. Foram avaliados dados sociodemográficos, obstétricos e variáveis relacionadas ao diagnóstico e tratamento da gestante e do parceiro. Apenas três (5,2\%; IC95\%: 1,8\%-14,1\%) gestantes foram consideradas adequadamente tratadas. O principal motivo da inadequação do tratamento foi a falta ou inadequação do tratamento do parceiro (88\% dos casos; IC95\%: 76,2\%-94,4\%). Foi possível constatar a necessidade de um segundo VDRL no terceiro trimestre de gestação. Os dados evidenciaram que o atendimento recebido pela gestante não foi suficiente para garantir o controle da sífilis congênita.

Sífilis Congênita; Gestantes; Cuidado Pré-Natal 


\section{Colaboradores}

A. L. A. Campos, M. A. L. Araújo e M. L. C. Gonçalves participaram da concepção, análise, redação e revisão final do artigo. S. P. Melo participou da concepção, análise e redação do manuscrito.

\section{Referências}

1. Secretaria de Vigilância em Saúde, Ministério da Saúde. Protocolo para a prevenção de transmissão vertical de HIV e sífilis - manual de bolso. Brasília: Ministério da Saúde; 2007.

2. Pires O, Pimentel ZNS, Santos MVS, Santos WA. Vigilância epidemiológica da sífilis na gravidez no centro de saúde do bairro Uruará-Área Verde. DST J Bras Doenças Sex Transm 2007; 19:162-5.

3. Schmid G. Economic and programmatic aspects of congenital syphilis prevention. Bull World Health Organ 2004; 82:402-9.

4. Secretaria de Vigilância em Saúde, Ministério da Saúde. Diretrizes para o controle da sífilis congênita. Brasília: Ministério da Saúde; 2005.

5. Galban E, Benzaken AS. Situación de la sífilis en 20 países de Latinoamérica y el Caribe: año 2006. DST J Bras Doenças Sex Transm 2007; 19:166-72.

6. Valderrama J, Zacarias F, Mazin R. Sífilis materna y sífilis congénita en América Latina: un problema grave de solución sencilla. Rev Panam Salud Pública 2004; 16:211-7.

7. Revollo R, Tinajeros F, Hilari C, Garcia SG, Zegarra L, Díaz-Olaviarreta C, et al. Sífilis materna y congénita en cuatro provincias de Bolivia. Salud Pública Méx 2007; 49:422-8.
8. Departamento de Ações Programáticas Estratégicas, Ministério da Saúde. Política nacional de atenção integral à saúde da mulher: princípios e diretrizes. Brasília: Ministério da Saúde; 2004.

9. Saraceni V, Guimarães MHFS, Theme Filha MM, Leal MC. Mortalidade perinatal por sífilis congênita: indicador da qualidade da atenção à mulher e à criança. Cad Saúde Pública 2005; 21:1244-50.

10. Wolf T, Shelton E, Sessions C, Miller T. Screening for syphilis infection in pregnant women: evidence for the U.S. Preventive Services Task Force Reaffirmation recommendation statement. Ann Intern Med 2009; 150:710-6.

11. Centers for Disease Control and Prevention. Congenital syphilis - United States, 2003-2008. MMWR Morbid Mortal Wkly Rep 2010; 59:413-7.

12. Secretaria de Vigilância em Saúde, Ministério da Saúde. Diretrizes para o controle da sífilis congênita - manual de bolso. Brasília: Ministério da Saúde; 2006.

13. Berman SM. Maternal syphilis: pathophysiology and treatment. Bull World Health Organ 2004; 82:433-8. 
14. Sá RAM, Bornia RBG, Cunha AA, Oliveira CA, Rocha GPG, Giordano EB. Sífilis e gravidez: avaliação da prevalência e fatores de risco nas gestantes atendidas na Maternidade Escola - UFRJ. DST J Bras Doenças Sex Transm 2001; 13:6-8.

15. Lima BGC, Costa MCN, Dourado MIC. Avaliação da qualidade do rastreamento de HIV/AIDS e sífilis na assistência pré-natal. Epidemiol Serv Saúde 2008; 17:123-7.

16. De Lorenzi DRS, Madi JM. Sífilis congênita como indicador de assistência pré-natal. Rev Bras Ginecol Obstet 2001; 23:647-52.

17. Secretaria de Vigilância em Saúde, Ministério da Saúde. Boletim epidemiológico - AIDS e DST. Brasília: Ministério da Saúde; 2007.

18. Figueiró-Filho EA, Gardenal RVC, Assunção LA, Costa GR, Periotto CRL, Vedovatte CA, et al. Sífilis congênita como fator de assistência pré-natal no Município de Campo Grande - MS. DST J Bras Doenças Sex Transm 2007; 19:139-43.

19. Almeida MFG, Pereira SM. Caracterização epidemiológica da sífilis congênita no Município de Salvador, Bahia. DST J Bras Doenças Sex Transm 2007; 19:144-56.

20. Secretaria de Políticas de Saúde, Ministério da Saúde. Implantação do programa de humanização no pré-natal e nascimento. Brasília: Ministério da Saúde; 2000

21. Puccini RF, Pedroso GC, Silva EMK, Araújo NN. Eqüidade na atenção pré-natal e ao parto na área da Região Metropolitana de São Paulo, 1996. Cad Saúde Pública 2003; 19:35-45.

22. Kilsztajn S, Rossbach A, Carmo MSN, Sugahara GTL. Assistência pré-natal, baixo peso e prematuridade no Estado de São Paulo, 2000. Rev Saúde Pública 2003; 37:303-10.

23. Secretaria da Vigilância em Saúde, Ministério da Saúde. Plano estratégico - Programa Nacional de DST e AIDS. Brasília: Ministério da Saúde; 2005.

24. Koffman MD, Bonadio IC. Avaliação da atenção pré-natal em uma instituição filantrópica da Cidade de São Paulo. Rev Bras Saúde Matern Infant 2005; 5 Suppl 1:523-32.
25. Serruya SJ, Cecatti JG, Lago TG. O Programa de Humanização no Pré-natal e Nascimento do Ministério da Saúde no Brasil: resultados iniciais. Cad Saúde Pública 2004; 20:1281-9.

26. Lima LHM, Viana MC. Prevalence and risk factors for HIV, syphilis, hepatitis B, hepatitis C, and HTLV-I/II infection in low-income postpartum and pregnant women in Greater Metropolitan Vitória, Espírito Santo State, Brazil. Cad Saúde Pública 2009; 25:668-76.

27. Araújo MAL, Silva DMA, Silva RM, Gonçalves MLC. Análise da qualidade dos registros nos prontuários de gestantes com exame de VDRL reagente. Rev APS 2008; 11:4-9.

28. Silveira DS, Santos IS, Costa JSD. Atenção pré-natal na rede básica: uma avaliação da estrutura e do processo. Cad Saúde Pública 2001; 17:131-9.

29. Trevisan MR, De Lorenzi DRS, Araújo NM, Khaddoun E. Perfil da assistência pré-natal entre usuárias do Sistema Único de Saúde em Caxias do Sul. Rev Bras Ginecol Obstet 2002; 24:293-9.

30. Saraceni V, Leal MC. Avaliação da efetividade das campanhas para eliminação da sífilis congênita na redução da morbi-mortalidade perinatal. Município do Rio de Janeiro, 1999-2000. Cad Saúde Pública 2003; 19:1341-9.

31. Lima BGC. Abordagem da sífilis como indicador de qualidade da assistência pré-natal em Salvador. Rev Baiana Saúde Pública 2004; 28:9-18.

32. Secretaria de Atenção à Saúde, Ministério da Saúde. Pré-natal e puerpério: atenção qualificada e humanizada - manual técnico. Brasília: Ministério da Saúde; 2005.

33. Rodrigues CS, Guimarães MDC, César CC. Missed opportunities for congenital syphilis and HIV perinatal transmission prevention. Rev Saúde Pública 2008; 42:851-8.

34. Donalísio MR, Freire JB, Mendes ET. Investigação da sífilis congênita na microrregião de Sumaré, Estado de São Paulo, Brasil - desvelando a fragilidade do cuidado à gestante e ao recém nascido. Epidemiol Serv Saúde 2007; 16:165-73.

Recebido em 11/Dez/2009

Versão final reapresentada em 19/Jul/2010

Aprovado em 26/Jul/2010 